for 30 weeks (treatment period 1). The primary endpoint was $a \geq 20 \%$ improvement in ACR response (ACR20) at Wk 14. At Wk 30 (treatment period 2 [TP2]), patients receiving IFX-EU were blindly re-randomised $(1: 1)$ to remain on IFX-EU or transition to GP1111 for 24 wks. Here we report longer-term efficacy, safety and immunogenicity data from Wks 30-54.

Results: 650 patients were randomised initially (GP1111, $\mathrm{n}=324$; IFX-EU, $\mathrm{n}=326$ ). At Wk 30, 566 patients entered TP2 (continued GP1111, $n=280$; continued IFX-EU, $n=143$; switched from IFX-EU to GP1111, $n=143$ ). ACR20 rates and DAS28-CRP scores were comparable between groups at all TP2 visits after rerandomisation in the TP2 population (figure 1). Incidences of TP2 treatment-emergent adverse events (AEs) $(36.8 \%, 33.6 \%$, and $37.8 \%)$, serious AEs $(4.6 \%, 7.7 \%$ and $2.8 \%)$ and infusion-related reactions (3.2\%, 8.4\% and $4.2 \%)$ were comparable between the GP1111/GP1111, IFX-EU/IFX-EU, and IFX-EU/GP1111 groups, respectively. Pre-dose ADA rates at Wk 30 (TP2) were $47.1 \%, 53.8 \%$ and $45.5 \%$ for the GP1111/GP1111, IFX-EU/IFX-EU, and IFX-EU/GP1111 groups, respectively. Overall, post-dose ADA rates in TP2 were comparable between groups $(52.1 \%, 60.1 \%$, and $58.0 \%$ respectively).
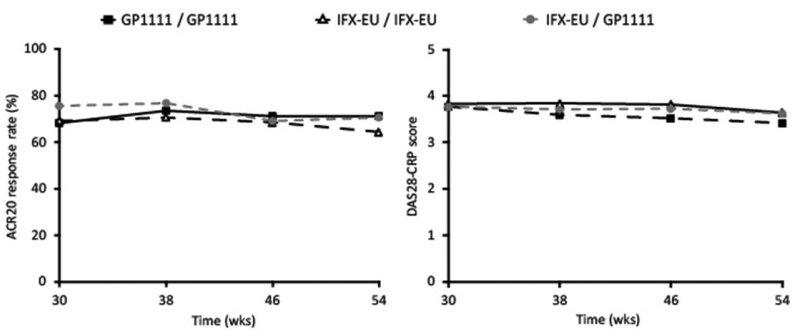

Abstract FRI0137 - Figure 1. ACR20 response rate and change in DAS28-CRP score at Wk 30 and 54 for the overall population during TP2

Conclusions: Results from TP2 (Wks 30-54) continued to show the absence of clinically meaningful differences in efficacy, safety and immunogenicity between patients with RA remaining on GP1111 or IFX-EU, or when blindly switched from IFX-EU to GP1111.

Disclosure of Interest: R. Alten Grant/research support from: Pfizer Inc., Consultant for: Pfizer Inc., Speakers bureau: Pfizer Inc., V. Tseluyko Speakers bureau: Pfizer Inc., AstraZeneca, Bayer, Boehringer Ingelheim, Servier, Sanofi, Takeda, KRKA, T. Hala: None declared, S. Mehmedagic: None declared, M. Pileckyte: None declared, E. Dokoupilová: None declared, D. Jovic: None declared, M. Rehman Shareholder of: Proctor and Gamble, Employee of: Pfizer Inc., M. Zhang Shareholder of: Pfizer Inc., Employee of: Pfizer Inc., L. Sewell Shareholder of: Pfizer Inc., Employee of: Pfizer Inc., S. Hackley Shareholder of: Pfizer Inc., Employee of: Pfizer Inc., S. Salts Shareholder of: Pfizer Inc., Mirati Therapeutics, Employee of: Pfizer Inc., C. Cronenberger Shareholder of: Pfizer Inc., Employee of: Pfizer Inc., K. Schumacher Shareholder of: Novartis, Employee of: Sandoz Biopharmaceuticals, O. von Richter Employee of: Sandoz Biopharmaceuticals, B. Batko Consultant for: Pfizer Inc., Sandoz, MSD

DOI: 10.1136/annrheumdis-2018-eular.5121

\section{FRI0138 TREAT TO TARGET STRATEGY PLUS CERTOLIZUMAB IN COMPARISON TO CONTINUED, FIXED CSDMARD PLUS CORTICOSTEROIDS IN PATIENTS WITH RHEUMATOID ARTHRITIS AND INADEQUATE RESPONSE TO CSDMARDS (REMISSION BY INTRA- ARTICULAR INJECTION PLUS CERTOLIZUMAB, THE RICE STUDY): A MULTI-CENTRE RANDOMISED CONTROLLED TRIAL}

$\underline{\text { R. Mueller }}{ }^{1}$, M. Spaeth ${ }^{2}$, C. von Restorff ${ }^{3}$, C. Ackermann ${ }^{4}$, J. von Kempis ${ }^{1}$ ${ }^{1}$ Department of Rheumatology, Kantonsspital St. Gallen, St. Gallen; ${ }^{2}$ Division of Rheumatology, Spital Linth, Uznach; ${ }^{3}$ Medical practice, Männedorf, Switzerland; ${ }^{4}$ Medical practice, Triesen, Liechtenstein

Background: Treatment of rheumatoid arthritis (RA) includes the use of conventional (cs) or targeted synthetic (ts) and biologic disease-modifying anti-rheumatic drugs (DMARDs) and oral or subcutaneous (SC) glucocorticoids (GC).

Objectives: We aimed to test the hypothesis that an improved outcome can be achieved by employing a treat to target (T2T) strategy optimising csDMARD, oral, and SC-GC treatment in parallel to a new onset certolizumab pegol (CZP) in RA patients with an incomplete response to csDMARD as compared to a conventional step up strategy with CZP

Methods: We designed a randomised controlled trial in four specialised rheumatological units. 43 patients with active RA ( $\geq 6$ tender, $\geq 6$ swollen joints, and ESR $\geq 20 \mathrm{~mm} / \mathrm{h}$ or $\mathrm{CRP} \geq 7 \mathrm{mg} / \mathrm{L}$ ) despite csDMARD treatment for $\geq 3$ months and naïve to biologic DMARDs were randomly allocated either to CZP plus a treat to target strategy (T2T group, $n=21$ ) or add on of CZP to a fixed dose of the already established csDMARD with or without established GCs (fixed dose group, $n=22$ ). Patients of both groups received $400 \mathrm{mg} \mathrm{CZP}$ at week 0,2 , and 4 (loading dose), followed by 200 mg every 2 weeks. The T2T strategy consisted in a step up in, or to, SC-methotrexate (dose: $15 \geq 20 \geq 25 \mathrm{mg} /$ week), followed by leflunomide $(20 \mathrm{mg} / \mathrm{d})$ and then by sulfasalazine $(2 \times 1000 \mathrm{mg} / \mathrm{d})$. In parallel, oral GCs were initiated in the T2T group at $20 \mathrm{mg} / \mathrm{d}$ and tapered every 5 days (15-12.5-10-7.55-2.5-0 mg/d). The decision to take the next step in the DMARD modification and addition of oral GCs was taken depending on the achievement of LDA (low disease activity: DAS28 <3.2) at the 4 -weekly visits. Injections of up to five tender and/or swollen joints with triamcinolone (small joints $10 \mathrm{mg}$, intermediate $20 \mathrm{mg}$ and big joints $40 \mathrm{mg}$; max. $100 \mathrm{mg} / \mathrm{visit}$ ) was allowed in the T2T group.

The primary outcome measure was ACR 50 response after 24 weeks. The analysis was intention-to-treat.

Results: Three patients dropped out during the study ( $\mathrm{n}=2$ T2T, $\mathrm{n}=1$ fixed dose). ACR 50 was achieved in 16 out 21 T2T patients (76.2\%) as compared to 8 out of 22 fixed dose patients (36.4\%; Chi $\left.^{2}: 5.355, \mathrm{p}=0.020\right)$. ACR 20 and 70 responses were achieved in $90.5 \%$ and $71.4 \%$ of the T2T patients and $59.1 \%$ and $27.3 \%$ of the fixed dose patients, respectively $(p=0.045$ and $p=0.010$, resp.). Mean reduction in DAS28 were significantly and HAQ-DI markedly greater in the T2T group than in the fixed dose group (DAS28: -3.9 [SD 1.2] vs. -2.2 [SD 1.5], $p<0.0006$ HAQ-DI: -0.63 [SD 0.58] vs. 0.20 [SD 0.67], $p<0.14$ ). All but five of the T2T patients required only one modification of cSDMARD and one additional course of oral GC. 10.2 joints (mean) were infiltrated with triamcinolone in the T2T group (av. dose $14.1 \mathrm{mg} /$ injection). The adverse event rate was similar for both groups (T2T $n=51$; fixed dose $n=55$ ).

Conclusions: Treat to target management with optimisation of csDMARDs, oral and intra-articular glucocorticoids of RA patients in parallel to additional CZP treatment was safe and substantially improves disease activity and the patient related outcome HAQ-DI in comparison to additional CZP to a fixed dose of csDMARDS. Disclosure of Interest: R. Mueller Grant/research support from: Unlimited grant by UCB pharmaceuticals, M. Spaeth: None declared, C. von Restorff: None declared, C. Ackermann: None declared, J. von Kempis: None declared DOI: 10.1136/annrheumdis-2018-eular.5556

\section{FRI0139 \\ COMPARATIVE SAFETY OF ABATACEPT IN RHEUMATOID ARTHRITIS WITH COPD: A REAL-WORLD POPULATION-BASED OBSERVATIONAL STUDY}

S. Suissa ${ }^{1}$, P. Ernst ${ }^{1}$, S. Dell'Aniello ${ }^{1}$, S. Shen ${ }^{2}$, T.A. Simon ${ }^{2} .{ }^{1} M c G i l l$ University, Montreal, Canada; ${ }^{2}$ Bristol-Myers Squibb, Princeton, USA

Background: In the ASSURE trial (NCT00048932) comparing abatacept with placebo for the treatment of RA, there was an increased incidence of respiratory serious adverse events (SAEs; COPD exacerbation/worsening, bronchitis and pneumonia) in those receiving abatacept among the subgroup of 54 patients with a history of chronic obstructive pulmonary disease (COPD). ${ }^{1}$

Objectives: To assess whether patients with RA and a history of co-morbid COPD treated with abatacept in a real-world, observational setting, have an increased risk of respiratory SAEs compared with similar patients treated with other biologic (b)DMARDs or the targeted synthetic DMARD tofacitinib (tofa).

Methods: The Truven MarketScan ${ }^{\oplus}$ Commercial and Supplemental Medicare databases were used to identify adult patients diagnosed with RA and COPD who were treated with abatacept, another bDMARD or tofa between January ${ }^{2007}$ and December 2015. Other bDMARDs included adalimumab, anakinra, certolizumab, etanercept, golimumab, infliximab, rituximab and tocilizumab. A prevalent newuser study cohort design ${ }^{2}$ was used in which each new user of abatacept was time- and propensity score-matched to two new users of other bDMARDs or tofa. Patients were required to have $\geq 6$ months of continuous health plan enrolment before cohort entry and were followed up until the end of enrolment in the database or 31 December 2015. Propensity scores of abatacept treatment were estimated from the baseline covariates using a conditional logistic regression mode separately in incident new users and prevalent new users. Patients with score ranges common to both abatacept and the comparator cohorts were included. An as-treated analysis based on the Cox proportional hazard regression model was used to estimate the hazard ratios (HRs) of respiratory SAEs associated with abatacept compared with other bDMARDs or tofa, further adjusted for confounders found to be unbalanced despite matching on propensity scores.

Results: A total of 9746 patients with RA and COPD initiated bDMARD or tofa therapy and included 1807 new users of abatacept matched to 3547 new users of another bDMARD or tofa. The matched cohort was followed for up to 9 years (mean 2.0 years); 53\% were incident users. For users of abatacept relative to other bDMARDs or tofa, the adjusted HRs $(95 \% \mathrm{Cl})$ of respiratory SAEs were: hospitalisation for COPD exacerbation: 0.57 (0.30, 1.05); hospitalisation for pneumonia/influenza: 1.39 (0.91, 2.12); outpatient pneumonia/influenza: 1.04 (0.85, 
1.28) and outpatient bronchitis: $0.80(0.57,1.14)$. Results remained unchanged with sensitivity analyses.

Conclusions: In this real-world study of patients with RA and COPD, the incidence of pre-specified respiratory SAEs was not significantly increased in patients using abatacept compared with those using other bDMARDs or tofa. The safety signal based on the subgroup of 54 patients from the ASSURE trial could not be confirmed in this large and long-term cohort study of over 5000 patients.

\section{REFERENCES:}

[1] Weinblatt E, et al. Arthritis Rheum 2006;54:2807-16.

[2] Suissa A, et al. Pharmacoepidemiol Drug Saf 2017;26:459-68.

Disclosure of Interest: S. Suissa Consultant for: Bristol-Myers Squibb, P. Ernst: None declared, S. Dell'Aniello: None declared, S. Shen Shareholder of: BristolMyers Squibb, Employee of: Bristol-Myers Squibb, T. A. Simon Shareholder of: Bristol-Myers Squibb, Employee of: Bristol-Myers Squibb

DOI: 10.1136/annrheumdis-2018-eular.1505

\section{FRI0140 INDIVIDUALISED INFLIXIMAB TREATMENT: A TREATMENT STRATEGY BASED ON THERAPEUTIC DRUG MONITORING}

S.W. Syversen ${ }^{1}$, G.L. Goll' ${ }^{1}$ K.K. Jørgensen ${ }^{2}, \varnothing$. Sandanger $^{3}$, J. Gehin ${ }^{4}$, C. Mørk ${ }^{5}$, T.K. Kvien ${ }^{1}$, J. Jahnsen ${ }^{2}$, N. Bolstad ${ }^{4}$, E.A. Haavardsholm ${ }^{1} .{ }^{1}$ Rheumatology, Diakonhjemmet, ${ }^{2}$ Gastroenterology, Akershus University Hospital; ${ }^{3}$ Dermatology; ${ }^{4}$ Laboratory Medicine, Oslo University Hospital, Oslo; ${ }^{5}$ Dermatology, None, Lørenskog, Norway

Background: Infliximab (INX) and other targeted therapies have greatly improved the treatment of patients with RA, SpA and PsA, but a significant proportion of patients either do not respond sufficiently to therapy or loose efficacy over time. Recent advances in assay development have revealed an extensive individual variation in serum drug concentrations suggesting both under- and overtreatment of a substantial proportion of patients. Many patients develop anti-drug antibodies (ADAb) during therapy, contributing to reduced drug levels, inefficacy and adverse events. Therapeutic drug monitoring (TDM), i.e. individual dose adjustments according to serum drug levels, can probably increase effectiveness of treatment with INX and other biological drugs.

Objectives: To develop an individualised treatment strategy based on TDM in order to optimise efficacy of INX treatment.

Methods: The treatment strategy has been developed by the steering committee of the NORwegian DRUg Monitoring study (NOR-DRUM), based on a systematic literature review (SLR), unpublished data and expert opinion. A SLR was performed in May 2016 to identify the therapeutic range. In Norway neutralising $A D A b$ are measured with an "in house" assay. For this assay, ADAb levels $>50$ $\mu \mathrm{g} / \mathrm{L}$ are defined as "high" leading to a recommendation to switch therapy. This cut-off is based on own s-INX and ADAb data (Diakonhjemmet Hospital during 2015-2016) and clinical experience. The proposed strategy has been developed through a series of meetings in the project group consisting of national leading experts in this field (both clinicians experienced with TDM and laboratory physicians) and with additional input from international key experts in the scientific advisory board of the NOR-DRUM study.

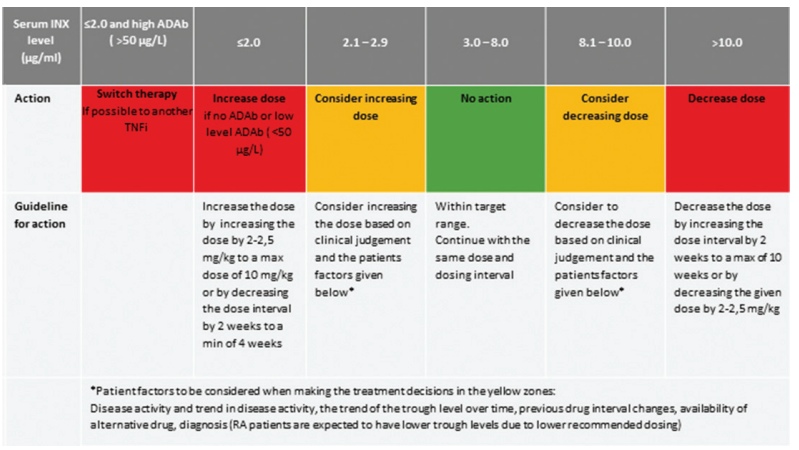

Results: The treatment strategy from infusion number 4 onwards is depicted in the figure 1. The therapeutic range for serum INX (through levels) is defined as 3$8 \mu \mathrm{g} / \mathrm{ml}$ (figure 1, green zone). During the induction phase (infusion 1-3) the recommendation is to keep the level $>20 \mu \mathrm{g} / \mathrm{ml}$ at infusion 2 and $>15 \mu \mathrm{g} / \mathrm{ml}$ at infusion 3. A guideline for action according to levels outside the therapeutic range is given in the figure 1. Dose modifications may be performed either as changes in doses or intervals as stated in the figure 1. If the patients develop high levels of $\mathrm{ADAb}$ the recommendation is to switch therapy.
Conclusions: An individualised treatment strategy based on TDM has the potential to optimise therapy with infliximab and other biological drugs by; 1) prevention of treatment failure by identification of patients with drug levels below the therapeutic range, 2) reduction of overtreatment, which predispose to side effects and increase costs, and 3) early identification of ADAb development, with the possibility to detect treatment failures prior to a clinical flare and to prevent hypersensitivity reactions. This approach has high face validity, and the effectiveness compared to regular care is being investigated in an ongoing randomised clinical trial, NOR-DRUM (NCT03074656).

Disclosure of Interest: S. Syversen Consultant for: Roche, G. Goll Consultant for: AbbVie, Biogen, Boehringer Ingelheim, Orion Pharma, Eli Lilly, Novartis, Pfizer, Roche, UCB, K. Jørgensen Consultant for: Tillott, Celltrion, Intercept, $\varnothing$ Sandanger: None declared, J. Gehin Consultant for: Roche, C. Mørk Consultant for: Abbvie, Novartis, LEO Pharma, ACI hud Norge, Cellegene AS, Galderma Nor$\operatorname{dic}$ AB, T. K. Kvien Consultant for: AbbVie, Biogen, BMS, Boehringer Ingelheim, Celgene, Celltrion, Eli Lilly, Epirus, Hospira, Merck-Serono, MSD, Mundipharma, Novartis, Oktal, Orion Pharma, Hospira/Pfizer, Roche, Sandoz and UCB, J. Jahnsen Consultant for: AbbVie, Celltrion, Takeda, Napp Pharm, AstroPharma, Hikma, Orion Pharma, Pfizer, N. Bolstad Consultant for: Pfizer, Roche, Orion Pharma, Napp pharm, Takeda, E. A. Haavardsholm Consultant for: AbbVie, Pfizer, MSD, Roche, UCB

DOI: 10.1136/annrheumdis-2018-eular.1433

\section{FRI0141 VACCINATION DECISIONS AND INCIDENCE OF NEONATAL INFECTIONS IN MOTHERS EXPOSED TO BIOLOGICALS DURING PREGNANCY}

S.J. Bond ${ }^{1,2}$, L. Young ${ }^{1,2}$, K. Poulsen ${ }^{2}$, H.L. Barrett ${ }^{3}$, A.L. Taylor ${ }^{4,5}$, C. Barrett ${ }^{1,2}$ ${ }^{1}$ University of Queensland; ${ }^{2}$ Metro North, Queensland Health; ${ }^{3}$ Mater Health, Brisbane; ${ }^{4}$ Royal Perth Hospital; ${ }^{5}$ University of Western Australia, Perth, Australia

Background: Rheumatologic diseases commonly affect women of childbearing age $\left(.^{1,2}\right.$ There is currently limited data available regarding the safety of vaccinations in infants after in utero exposure to biologics.

Objectives: To determine the vaccination decisions of mothers with rheumatologic diseases exposed to biologics during pregnancy and the incidence of serious neonatal infections after third trimester exposure.

Methods: All Australian women with inflammatory arthritis, exposed to biologics during the preconception, antenatal and/or postpartum periods, were invited to participate in the Pregnancy Exposed to Biological (PEB) study from May 2009 - . Jan 2018 Recruitment was via direct invitation from patients treating rheumatologists, community groups, and via social media. Following self-referral to the study, retrospective data was collected, including biological exposure, vaccination history and the incidence of serious neonatal infections, defined as infection requiring hospitalisation.

Results: Preliminary data is available regarding 35 offspring from 28 mothers. 34 of 35 offspring were vaccinated. 29 received vaccinations in accordance with the Australian National Immunisation Program Schedule. 1 mother declined to immunise her infant due to personal preference.

13 infants were exposed to a tumour necrosis factor inhibitor (TNFi) during the third trimester. Of these, 4 had Rotavirus vaccine delayed from 2 to 4 months and 1 infant until 6 months. 1 infant did not receive the Rotavirus vaccine at 2 months due to exposure to a TNFi while breastfeeding. There were no incidences of serious neonatal infections.

Conclusions: Current guidelines recommend deferring live vaccines, such as rotavirus, until after 6 months if exposed to a biologic in the third trimesterr. $\left({ }^{2-5}\right.$ Compliance with these recommendations was only observed in one infant in our study. One infant received delayed Rotavirus vaccination due to concern about TNFi exposure during breastfeeding; this is not in keeping with current guidelines. Of the 12 infants exposed to a biologic during the third trimester who did not delay live vaccination until after 6 months, there were no incidents of serious neonatal infections, in keeping with the findings of current published case series.

\section{REFERENCES}

[1] Brouwer J, Hazes JM, Laven JS, Dolhain RJ. Fertility in women with rheumatoid arthritis: influence of disease activity and medication. Ann Rheum Dis. 2015;74(10):1836-41.

[2] Gotestam Skorpen C, Hoeltzenbein M, Tincani A, Fischer-Betz R, Elefant E, Chambers C, et al. The EULAR points to consider for use of antirheumatic drugs before pregnancy, and during pregnancy and lactation. Ann Rheum Dis. 2016;75(5):795-810.

[3] Flint J, Panchal S, Hurrell A, van de Venne M, Gayed M, Schreiber K, et al. BSR and BHPR guideline on prescribing drugs in pregnancy and breastfeeding-Part I: standard and biologic disease modifying anti-rheu matic drugs and corticosteroids. Rheumatology (Oxford). 2016;55(9):16937.

[4] Health AGDo. 3.3 Groups with special vaccination requirements 2017. 